\title{
EMBEDDING OF THE DUNCE HAT
}

\author{
J. KRASINKIEWICZ AND S. SPIEŻ
}

(Communicated by Alexander N. Dranishnikov)

\begin{abstract}
In this note we show that the famous Borsuk contractible noncollapsible 2-polyhedron, generally known as the dunce hat, does not embed in any product of two curves but quasi-embeds in the "three-page book".
\end{abstract}

All spaces discussed in this paper are assumed to be metrizable and all mappings (also called maps) are continuous. By a compactum we mean a compact (metric) space, by a continuum we mean a non-void connected compactum, and by a curve we mean a 1-dimensional continuum. All polyhedra are compact.

By $\mu$ we denote the Menger curve. It is well known that if a compactum $X$ quasi-embeds in $\mu$, then $X$ embeds in $\mu$. (A metric space $X$ is said to quasi-embed in $Y$ if for each $\varepsilon>0$ there is an $\varepsilon$-mapping $f: X \rightarrow Y$.) In 3 the authors asked the following question about a possible extension of this result to finite products of copies of the Menger curve:

\section{Suppose $X$ quasi-embeds in $\mu^{n}$. Does $X$ embed in $\mu^{n}$ ?}

To our surprise, this question has been answered in the negative in a recent joint paper by S. A. Melikhov and J. Zając [6]. Actually, they proved that the Sklyarenko absolute retract 8 quasi-embeds in a product of two dendrites but does not embed in any product of two curves. The problem was left open for cases where $X$ is less complicated, for instance, for $X$ being a polyhedron. (Sklyarenko's example is not a polyhedron.)

The purpose of this note is to show that even for polyhedra the answer is negative. In fact, we are going to prove that the famous Borsuk contractible non-collapsible 2-dimensional polyhedron 1] (generally known as the dunce hat (cf. 9]); also called the Borsuk tube by Polish topologists (cf. 2])) is such a counterexample. In other words, we shall prove the following.

Theorem. The dunce hat does not embed in any product of two curves but quasiembeds in the "three-page book" $T \times I$.

By $T$ we denote the simple triod, and by $I$ we denote the unit interval, $I=[0,1]$.

First we recall a description of the Borsuk tube, slightly modifying the original description of Borsuk 1 . Let $\mathbb{B}^{2}$ denote the unit disc in the complex plane $\mathbb{C}$, and let $\mathbb{S}^{1}$ denote its boundary, the unit circle in $\mathbb{C}$. We define the Borsuk tube to be

Received by the editors June 27, 2011 and, in revised form, October 11, 2011.

2010 Mathematics Subject Classification. Primary 54C25; Secondary 54E45, 54F45, 55M10, $57 \mathrm{~N} 35$.

Key words and phrases. Embeddings, quasi-embeddings, Borsuk's example.

${ }^{1} \mathrm{~A}$ mapping $f: X \rightarrow Y$ is said to be an $\varepsilon$-mapping if diam $f^{-1}(y)<\varepsilon$ for each $y \in f(X)$. 

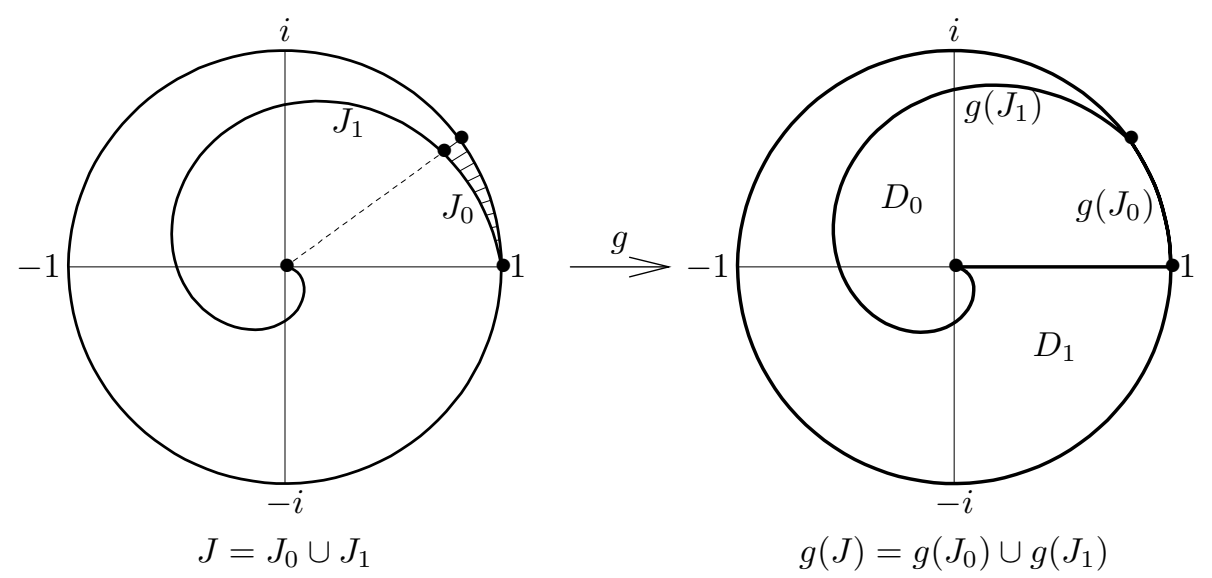

FIGURE 1

the quotient $B=\mathbb{B}^{2} / \sim$, where $(1-t) \exp (2 \pi i t) \sim \exp (2 \pi i t)$ for each $t \in I$. (The interval $I$ is a subset of $\mathbb{B}^{2}$ because the reals are regarded to be a subset of $\mathbb{C}$.) Thus $B$ is the quotient of $\mathbb{B}^{2}$ obtained by identifying the arc $J=\{(1-t) \exp (2 \pi i t): t \in I\}$ with $\mathbb{S}^{1}$; see Figure 1. (In the original description, Borsuk identifies $t \in I$ with $\exp (2 \pi i t) \in \mathbb{S}^{1}$ for each $t \in I$.) By its basic property $B$ is a contractible but not collapsible 2-polyhedron ([1]; cf. [9]). One easily verifies that $B$ is a quasi-2manifold 2 Hence by the Structure Theorem in 4 , we infer that $B$ does not embed in any product of two curves; see [4, Corollary 5.4. This proves the first assertion of our theorem. To complete the proof it remains to establish the second one.

From the definition it follows that $B$ is a compact metrizable space; let $d$ denote a fixed metric on $B$. Fix a number $\epsilon>0$. Hence it remains to construct an $\epsilon$ mapping $f: B \rightarrow T \times I$. Let $q: \mathbb{B}^{2} \rightarrow B$ denote the quotient mapping. Observe that there is a number $a>0$ such that for all $z, z^{\prime} \in \mathbb{B}^{2}$ we have

(1) $\left|z-z^{\prime}\right|<a \Rightarrow d\left(q(z), q\left(z^{\prime}\right)\right)<\epsilon$.We may also assume that $a<1$. Then it is easy to define an equicontinuous family of mappings $g_{t}:(I, 0,1) \rightarrow(I, 0,1), t \in I$, satisfying the conditions

(2) $g_{0}=g_{1}=i d_{I}$,

(3) $g_{t}$ is a relative homeomorphism $(I,[1-t, 1]) \rightarrow(I,\{1\})$ for each $0 \leq t \leq a$,

(4) $g_{t}$ is a homeomorphism for each $a<t \leq 13$

These mappings define a mapping $g: \mathbb{B}^{2} \rightarrow \mathbb{B}^{2}$ given by the formula

$$
g(s \cdot \exp (2 \pi i t))=g_{t}(s) \cdot \exp (2 \pi i t)
$$

for each $s, t \in I$.

\footnotetext{
${ }^{2}$ A 2-dimensional compactum is called a quasi-2-manifold if each point $x \in X$ admits an open neighborhood $U$ such that every closed set $F$ separating $X$ between $x$ and $X \backslash U$ admits an essential map into $\mathbb{S}^{1}$.

${ }^{3}$ For instance, let us define: (i) if $t \in[0, a]$ put $g_{t}(s)=\frac{s}{1-t}$ for $s \leq 1-t$ and $g_{t}(s)=1$ for $s \geq 1-t$; (ii) if $t \in(a, 1]$ put $g_{t}(s)=\left(\frac{1-t}{(1-a)^{2}}+\frac{t-a}{1-a}\right) s$ for $s \leq 1-a$ and $g_{t}(s)=\frac{t-a}{1-a} s+\frac{1-t}{1-a}$ for $s>1-a$. Notice that each $g_{t}$ is composed of two linear maps defined on adjacent (possibly degenerate) subintervals of $I$.
} 
Then one can define another quotient space $B^{\prime}$ of $\mathbb{B}^{2}$ analogous to $B$, identifying the $\operatorname{arc} g(J)$ with $\mathbb{S}^{1}$; precisely, $B^{\prime}=\mathbb{B}^{2} / \sim^{\prime}$, where $g((1-t) \exp (2 \pi i t)) \sim^{\prime} \exp (2 \pi i t)$ for each $t \in I$. Let $q^{\prime}: \mathbb{B}^{2} \rightarrow B^{\prime}$ denote the quotient mapping. Since $g$ preserves the identifications, there exists a mapping $g^{\prime}: B \rightarrow B^{\prime}$ such that $q^{\prime} \circ g=g^{\prime} \circ q$; that is, the following diagram commutes:

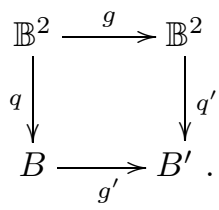

Then

(5) $g^{\prime}$ is an $\epsilon$-mapping.

Indeed, suppose $g^{\prime}$ sends two different points $q(z), q\left(z^{\prime}\right)$ of $B$ to the same point of $B^{\prime}$. Then $z, z^{\prime}$ do not lie in the same fiber of $q$. Since $g(z), g\left(z^{\prime}\right)$ lie in the same fiber of $q^{\prime}$, it follows that both $z, z^{\prime}$ lie in the same fiber of $g$, i.e. $z, z^{\prime} \in[1-t, 1] \exp (2 \pi i t)$ for some $t \in[0, a]$. Therefore, $\left|z-z^{\prime}\right|<a$; hence $d\left(q(z), q\left(z^{\prime}\right)\right)<\epsilon$ by (1). This proves (5).

Notice that $J$ is the union of two subarcs $J_{0}=\{(1-t) \exp (2 \pi i t): t \in[0, a]\}$ and $J_{1}=\{(1-t) \exp (2 \pi i t): t \in[a, 1]\}$. Likewise, $\mathbb{S}^{1}$ is the union of two arcs $S_{0}=\{\exp (2 \pi i t): t \in[0, a]\}$ and $S_{1}=\{\exp (2 \pi i t): t \in[a, 1]\}$. Moreover, we have: $g(J)$ is the union of two subarcs $g\left(J_{0}\right)$ and $g\left(J_{1}\right), g\left(J_{0}\right)=S_{0}$ and $g\left(J_{1}\right)$ meets $S_{1}$ at point $\exp (2 \pi i a)$ which is a common endpoint of these arcs, and off that point $g\left(J_{1}\right)$ lies in the interior of $\mathbb{B}^{2}$. It follows that $B^{\prime}$ is the quotient of $\mathbb{B}^{2}$ obtained by identifying the $\operatorname{arcs} g\left(J_{1}\right)$ and $S_{1}$. Therefore, $B^{\prime}$ is a collapsible 2-polyhedron and $q^{\prime}\left(S_{0}\right)$ is a free face of $B^{\prime}$ (i.e. each interior point $x$ of $q^{\prime}\left(S_{0}\right)$ admits a neighborhood that is a closed 2-disc with $x$ lying on its boundary). To complete the proof it suffices to show that $B^{\prime}$ embeds in $T \times I$.

To this end, present $B^{\prime}$ as a union of two sets $q^{\prime}\left(D_{0}\right)$ and $q^{\prime}\left(D_{1}\right)$, where $D_{0}$ is the disc bounded by the $\operatorname{arcs} I, S_{0}$ and $g\left(J_{1}\right)$, and $D_{1}$ is the disc bounded by $I, S_{1}$ and $g\left(J_{1}\right)$. Clearly, $q^{\prime}\left(D_{1}\right)$ is a disc and the circle $q^{\prime}(I)$ is its boundary. On the other hand, $q^{\prime}\left(D_{0}\right)$ meets $q^{\prime}\left(D_{1}\right)$ along the union $q^{\prime}\left(g\left(J_{1}\right) \cup I\right)$. Since the arc $q^{\prime}\left(g\left(J_{1}\right)\right)$ off its endpoint $q^{\prime}(0)$ lies entirely in the interior of the disc $q^{\prime}\left(D_{1}\right)$ and $q^{\prime}(I)$ is the boundary of that disc, one easily sees that $B^{\prime}$ embeds in $T \times I$.

Lemma. The suspension of a starlike compact set $X$ lying in $\mathbb{R}^{n}$ embeds in the product $X \times I$.

To prove this lemma we may assume that $X$ is starlike with respect to the point $\mathbf{0} \in \mathbb{R}^{n}$ and that $\mathbb{R}^{n+1}=\mathbb{R}^{n} \times \mathbb{R}$. Identify $X$ with $X \times\{0\}$. Then the suspension is the union of two cones over $X$ with vertices $(\mathbf{0},-1)$ and $(\mathbf{0}, 1)$. In this setting, the suspension is a subset of $X \times[-1,1]$.

Applying our theorem and this lemma we get the following.

Corollary. For each $n \geq 2$ there is an n-dimensional contractible polyhedron $P$ not embeddable in any product of $n$ curves but quasi-embeddable in a product of $n$ triods. Moreover, if $n=2 k$, then $P$ quasi-embeds in $T^{k} \times I^{k}$, and if $n=2 k+1$, then $P$ quasi-embeds in $T^{k} \times I^{k+1}$. 
Proof. Actually, we shall show that for $P$ we can take either the product $B^{k}=$ $B \times \ldots \times B$ if $n=2 k$, or the suspension $\sum B^{k}$ if $n=2 k+1$. In fact, the product $B^{k}$ of $k$ copies of the Borsuk tube is a quasi- $2 k$-manifold that is a product of quasi-2-manifolds; see 4, Corollary 2.3. Since $B^{k}$ is contractible, by the Structure Theorem 5.1 of [4, it does not embed in any product of $2 k$ curves. Since $B$ quasiembeds in $T \times I, B^{k}$ quasi-embeds in $(T \times I)^{k}=T^{k} \times I^{k}$. On the other hand, by Corollary 2.3 of [4, $\sum B^{k}$ is a quasi- $(2 k+1)$-manifold off two points (cf. the proof of Theorem 1.3 in [5]). Notice that $\sum B^{k}$ is contractible. Hence, by the Second Structure Theorem 6.1 of $[5], \sum B^{k}$ does not embed in any product of $2 k+1$ curves. Moreover, $\sum B^{k}$ quasi-embeds in $\sum(T \times I)^{k}$, hence quasi-embeds in $(T \times I)^{k} \times I=T^{k} \times I^{k+1}$, by the above lemma and the starlikeness of $(T \times I)^{k}$ in $\mathbb{R}^{3 k}$

\section{PROBLEMS}

Problem 1. Characterize 2-dimensional polyhedra quasi-embeddable in a product of two curves.

Problem 2. Does the "Bing house" quasi-embed in a product of two curves?

The "Bing house" is another example of a contractible non-collapsible 2-polyhedron [7. By the same argument as in the case of the Borsuk tube, it does not embed in any product of two curves.

Problem 3. Does the suspension $\Sigma^{n} B, n>1$, embed in a product of $n+2$ curves?

Notice that by the Structure Lemma 4.8 in $4, \Sigma^{n} B$ does not embed in any product of $n+2$ graphs. Therefore, a positive solution of the problem from [5] implies a negative solution to Problem 3 above. Also notice that by our lemma, $\Sigma^{n} B$ quasi-embeds in $T \times I^{n+1}$ because $B$ quasi-embeds in $T \times I$.

\section{ACKNOWLEDGMENT}

The authors thank Dr. M. Sobolewski for his valuable remarks.

\section{REFERENCES}

[1] K. Borsuk, Über das Phaenomen der Unzerlegbarkeit in der Polyedertopologie, Comment. Math. Helv. 8 (1935), 142-148. MR1509522

[2] Collected papers. PWN-Polish Scientific Publishers, Warsaw, 1983. MR0716862 (85e:01058)

[3] A. Koyama, J. Krasinkiewicz and S. Spież, Embedding compacta into products of curves. arXiv: 0712.3470v1 [math.GT] 20 Dec 2007, pp. 1-71.

[4] _ Generalized manifolds in products of curves. Trans. Amer. Math. Soc. 363 (2011), no. 3, 1509-1532. MR2737275

[5] J. Krasinkiewicz and S. Spież, Extension of the Borsuk theorem on non-embeddability of spheres. Proc. Amer. Math. Soc. 140 (2012), 4035-4040. MR2944743

[6] S. A. Melikhov and J. Zając, Contractible polyhedra in products of trees and absolute retracts in products of dendrites, preprint.

[7] C. P. Rourke and B. J. Sanderson, Introduction to piecewise-linear topology. Springer-Verlag, Berlin, Heidelberg, New York, 1982. MR665919 (83g:57009) 
[8] E. G. Sklyarenko, On homologically locally connected spaces. Izv. Akad. Nauk SSSR Ser. Mat. 44 (1980), no. 6, 1417-1433, 1440; English transl., Math. USSR - Izvestiya 17 (1981), no. 3, 601-614. MR603583 (82e:55011)

[9] E. C. Zeeman, On the dunce hat. Topology 2 (1964), 341-358. MR0156351 (27:6275)

Institute of Mathematics, Polish Academy of Sciences, ul. Śniadeckich 8, 00-956, WARSAW, POLAND

E-mail address: jokra@impan.pl

Institute of Mathematics, Polish Academy of Sciences, ul. Śniadeckich 8, 00-956, WARSAW, POLAND

E-mail address: spiez@impan.pl 